\title{
European option pricing model with generalized Ornstein-Uhlenbeck process under stochastic earning yield and stochastic dividend yield
}

\author{
N. Phewchean ${ }^{1,2^{*}}$ (D) and Y. Wu ${ }^{3}$
}

\section{${ }^{*}$ Correspondence:}

nattakorn.phe@mahidol.ac.th

${ }^{1}$ Department of Mathematics,

Faculty of Science, Mahidol

University, Bangkok, Thailand

${ }^{2}$ Centre of Excellence in

Mathematics, CHE, Bangkok,

Thailand

Full list of author information is

available at the end of the article

\section{空 Springer}

\begin{abstract}
This paper aims to examine and establish the models for European option pricing which include parameters of stochastic dividend yield and stochastic earning yield. We generalize the Ornstein-Uhlenbeck process and define it as generalized Ornstein-Uhlenbeck process. We have learned that the firm stocks, according to Black-Scholes-Merton structure, obey the geometric Brownian motion process. Under a stochastic earning yield, the dividend yield complies with the generalized Ornstein-Uhlenbeck process. The firm dividend randomly deviates from the earning yield flow because of the presence of stochastic components of dynamic Wiener process of generalized Ornstein-Uhlenbeck. In this study, we model the stock price with stochastic earning yield, and stochastic dividend yield to be taking account stochastic market price of risk parameter which is mean-reverting as well. We developed explicit formulae for European call option pricing calculations. From numerical simulation, we could evaluate the performance of our new model that could be compared with other notable option pricing models by using actual option price data. The outcomes prove that our new model performance is best when compared with others.
\end{abstract}

MSC: $93 \mathrm{E} 03 ; 60 \mathrm{H} 10$

Keywords: Options pricing; Stochastic earning yield; Stochastic dividend yield; Black-Scholes-Merton model; Generalized Ornstein-Uhlenbeck process

\section{Introduction}

When making an investment decision, traders seek confidence in profit making. They consider a derivative financial instrument: an option. In Black-Scholes-Merton structure, many parameters are used to determine option price, such as dividend yield, volatility, interest rates, and time maturity [1]. However, this option pricing model leaves out some parameters and therefore does not reflect real world finance situation. Thus, the stochastic parameters should be taken into the calculation of the option valuation model. It can be seen that dividend yield is considered an important parameter that mainly makes an impact on the option pricing models [2-4]. In the development of option pricing model,

(c) The Author(s) 2019. This article is distributed under the terms of the Creative Commons Attribution 4.0 International License (http://creativecommons.org/licenses/by/4.0/), which permits unrestricted use, distribution, and reproduction in any medium, provided you give appropriate credit to the original author(s) and the source, provide a link to the Creative Commons license, and indicate if changes were made. 
an Ornstein-Uhlenbeck process is conducted in the pricing model mathematically to calculate the value of option [5-7].

In finance, both $\mathrm{P} / \mathrm{E}$ ratio and dividend yield are the common information that can be retrieved from our daily newspapers. Hence, we extend the Black-Scholes-Merton concept by including the P/E ratio into the model. Here, the earning yield is a critical factor in the dividend yield model. We set up stochastic differential equations to explain the stock pricing situation by taking into consideration stochastic dividend yield. Generalized process of Ornstein-Uhlenbeck is defined in order to explain option pricing models when stochastic earning yield is an element of stochastic dividend yield. Inspired by the approach used by Abraham Lioui [5], we characterize a Wiener process by generalizing the economic situation with $n$ risk parameters and explicitly obtain pricing formulae of an option of European call. We can then provide the option pricing formulae taking into account stochastic earning yield.

\section{The setting}

\subsection{The definitions}

A Wiener process is a random walk process in a continuous time. The OrnsteinUhlenbeck process is developed by having a character that the walk has the ability to move back to a central value or mean reverting property.

Over the past years, option valuation models have utilized the Ornstein-Uhlenbeck process which is an important main component for any economic study of valuation with stochastic property $[2,5,8]$. Such a model is commonly used to describe the stochastic behavior of many crucial variables in the real world of financial market such as interest rates, dividends, volatility, currency exchange rates, and commodity prices [9].

Definition 1 A stochastic process $x(t)$ is defined as the Ornstein-Uhlenbeck process:

$$
d x(t)=v(\mu-x(t)) d t+\sigma d W(t))
$$

where $W(t)$ is a Wiener process on $t \in[0, \infty)$ and $v>0, \sigma>0$, and $\mu$ are constant parameters.

As it is possible that a parameter can be dependent on itself or on other parameters or both, in this study, we assume that the stochastic process emulates the OrnsteinUhlenbeck process which may be a part of other Ornstein-Uhlenbeck processes. Generally, it clarifies a concept of, for example, a movement of particles with friction. In situation like this, a process $X(t)$, defined as a generalized Ornstein-Uhlenbeck process, may be useful in explaining such situations.

Definition 2 A stochastic process $X(t)$ is defined as the following generalized OrnsteinUhlenbeck process:

$$
\begin{aligned}
d X(t)= & v_{0}\left(\mu_{0}-X(t)\right) d t \\
& +v_{1}\left(\mu_{1}-Y_{1}(t)\right) d t+v_{2}\left(\mu_{2}-Y_{2}(t)\right) d t+\cdots+v_{n}\left(\mu_{n}-Y_{n}(t)\right) d t \\
& \left.+\sigma_{0} d W_{0}(t)+\sigma_{1} d W_{1}(t)+\sigma_{2} d W_{2}(t)+\cdots+\sigma_{n} d W_{n}(t)\right),
\end{aligned}
$$


where $Y_{i}(t)$ denotes the generalized Ornstein-Uhlenbeck process, $W(t)$ is the Wiener process, on $t \in[0, \infty)$ and parameters $v_{0}>0, \mu_{0}>0, \sigma_{0}>0, v_{i} \geq 0, \sigma_{i} \geq 0$, and $\mu_{i} \in \mathbb{R}$, where $i=1,2, \ldots, n$ are all constant.

We have an assumption that the precarious component is the risk factor that can impact on the aforementioned underlying asset, for example, a stock price can be affected by the market price of risks under the martingale measure $Q$. In this case, we can define market price of risks in a form of generalized Ornstein-Uhlenbeck.

Definition 3 The generalized Ornstein-Uhlenbeck Wiener process is defined as

$$
\widehat{W}(t)=W(t)+\int_{0}^{t} v_{1}(s) d s+\int_{0}^{t} v_{2}(s) d s+\cdots+\int_{0}^{t} v_{n}(s) d s,
$$

where $W(t)$ is the Wiener process and $v_{i}(t)$ is the generalized Ornstein-Uhlenbeck process when $i=1,2, \ldots, n$.

The objective of this study is to form an option pricing model on the basis of real financial situations. Every stochastic process and important condition will be taken into consideration and will be included in the mathematical model. Thus, we define the process as a process of generalized Ornstein-Uhlenbeck for the benefit of general situation financial model construction.

Definition 4 The generalized $X(t)$ is defined as a generalized Ornstein-Uhlenbeck process:

$$
\begin{aligned}
d \widehat{X}(t)= & v_{0}\left(\mu_{0}-X(t)\right) d t+v_{1}\left(\mu_{1}-\widehat{Y}_{1}(t)\right) d t+\cdots+v_{n}\left(\mu_{n}-\widehat{Y}_{n}(t)\right) d t \\
& \left.+\sigma_{0} d \widehat{W}_{0}(t)+\sigma_{1} d \widehat{W}_{1}(t)+\cdots+\sigma_{n} d \widehat{W}_{n}(t)\right),
\end{aligned}
$$

where $\widehat{Y}_{i}(t)$ is the generalized Ornstein-Uhlenbeck process, $\widehat{W}(t)$ is the generalized Ornstein-Uhlenbeck Wiener process, on $t \in[0, \infty)$ and parameters $\nu_{0}>0, \mu_{0}>0, \sigma_{0}>0$, $v_{i} \geq 0, \sigma_{i} \geq 0$, and $\mu_{i} \in \mathbb{R}$, where $i=1,2, \ldots, n$ are all constant.

In the following section, with all four definitions provided, we will illustrate the construction of the pricing models, under market price of risks, that includes proposed parameters, stochastic earning yield, and stochastic dividend yield.

\subsection{The models}

When developing the option pricing models, we assume the financial market to be completed with no arbitrage opportunity. The pricing probability space is $(\Omega, \mathbb{P}, \mathbb{F})$ where $\Omega$ is the pricing space with probability measure $\mathbb{P}$ and measurable events $\mathbb{F}$. A fixed martingale measure $\mathbb{Q}$ also exists. We assume $\mathbb{Q}$ to be equal to the probability measure $\mathbb{P}$ and dividends become martingale. We make such an assumption to guarantee no arbitrage opportunity in the market $[10,11]$. The stochastic processes in any pricing nature are adjusted to the filtration $\left\{\mathcal{F}_{t}\right\}$ provided by the Wiener process. It can be defined that $\mathcal{F}_{t}$-adapted processes $v_{1}(t), v_{2}(t)$, and $v_{3}(t)$ are multiple Brownian motions, according to 
Girsanov's theorem. From the Radon-Nikodym derivative equation, the martingale measure and measurable probability with multiple Brownian motions can be defined as follows [12]:

$$
\begin{aligned}
\left.\frac{d \mathbb{Q}}{d \mathbb{P}}\right|_{\mathcal{F}_{t}}= & \exp \left(-\int_{0}^{t} v_{1}(s) d W_{1}(s)-\int_{0}^{t} v_{2}(s) d W_{2}(s)-\int_{0}^{t} v_{3}(s) d W_{3}(s)\right. \\
& \left.-\frac{1}{2} \int_{0}^{t}\left(v_{1}(s)^{2}+v_{2}(s)^{2}+v_{3}(s)^{2}\right) d s\right), \quad \mathbb{P} \text {-a.s. }
\end{aligned}
$$

The Brownian motions $d W_{1}(s), d W_{2}(s)$, and $d W_{3}(s)$ are uncorrelated Wiener processes in one dimension which is described on a probability space $(\Omega, \mathbb{P}, \mathbb{F})$.

The prices of risky assets agree to a process of stocks which can be modeled as follows:

$$
\frac{d S(t)}{S(t)}=\left(\mu_{S}(t, S(t), \delta(t))-\delta(t)\right) d t+\sigma_{S} d W_{1}(t)
$$

where $S(0)>0$ and $\sigma_{S}$ is a positive constant of volatility.

As we consider the $\mathrm{P} / \mathrm{E}$ ratio to be related to the model of option pricing in this paper, the dividend yield is a component to determine the $\mathrm{P} / \mathrm{E}$ ratio.

$$
\begin{aligned}
\delta(t) & \approx \frac{1}{P / E} \cdot\left(\frac{\text { Dividend }}{\text { Earning Per Share }}\right) \\
& =(\text { Earning Yield }) \cdot\left(\frac{\text { Dividend }}{\text { Earning Per Share }}\right) .
\end{aligned}
$$

From this, we can presume the derivative of the dividend yield to depend on the earning yield instead. The dividend model is proposed as follows.

$$
\begin{aligned}
d \delta(t)= & \theta_{\delta}\left(\mu_{\delta}-\delta(t)\right) d t+\theta_{\psi}\left(\mu_{\psi}-\psi(t)\right) d t \\
& +\left(\sigma_{\delta 1} d W_{1}(t)+\sigma_{\delta 2} d W_{2}(t)+\sigma_{\delta 3} d W_{3}(t)\right),
\end{aligned}
$$

where $\delta(0)=0$ and parameters $\theta_{\delta}, \mu_{\delta}, \theta_{\psi}, \mu_{\psi}$, and $\sigma_{\delta i}$ are all constants when $i=1,2,3$.

As for earning yield $\psi(t)$, we define it by the random fluctuations under the generalized Ornstein-Uhlenbeck process as follows:

$$
d \psi(t)=\theta_{\psi}\left(\mu_{\psi}-\psi(t)\right) d t+\sigma_{\psi 1} d W_{1}(t)+\sigma_{\psi 2} d W_{2}(t)+\sigma_{\psi 3} d W_{3}(t)
$$

where setting $\psi(0)=0$ and parameters $\theta_{\psi}, \mu_{\psi}$, and $\sigma_{\psi i}$ are all constants when $i=1,2,3$.

It is to be noted that equation (8) can be applied to (7) and (7) is applied to (6). These two conditions form the relationship among the related stochastic functions. In certain cases, we can identify the correlation between two or more processes by letting $\sigma_{\delta i}$ and/or $\sigma_{\psi i}$ be equal to zero.

$v_{i}$ is the market price of risk (MPR) parameter by Definition 3 and is presumed to fit the generalized Ornstein-Uhlenbeck process as follows.

$$
d v_{i}(t)=\theta_{v i}\left(\mu_{v i}-v_{i}(t)\right) d t+\sigma_{v i} d W_{i}(t)
$$

where constants $v(0)=0, \theta_{v_{i}}, \mu_{v_{i}}$, and $\sigma_{v_{i}}$ are all positive when $i=1,2,3$. 
However, the MPR poses a consequence on the models in (6), (8), (9), and (10); therefore, we remodel the pricing formulae so that the risk factors are capsulated, which is beneficial to asset pricing in the real world.

\section{The method}

By applying Abraham Lioui's analytic method [5], assuming arbitrage-free, we can develop analytic proofs for European options formulas.

Considering the MPR, $v_{i}(t)$, the kernel of movement is changed, the result is that the generalized Ornstein-Uhlenbeck Wiener process $\widehat{W}_{i}(t)$ with MPR replaces the Wiener processes $W_{i}(t)$ of models (6)-(9). Once we get the risk movement parameter MPR, we apply Definition 3, and the general Wiener process can be defined as follows:

$$
\widehat{W}_{i}(t)=W_{i}(t)+\int_{0}^{t} v_{i}(s) d s .
$$

When models (6)-(9) have been adapted, the equation for calculating the stock price is

$$
\frac{d S(t)}{S(t)}=\left(\mu_{S}(t, S(t), \delta(t))-\delta(t)\right) d t+\sigma_{S} d \widehat{W}_{1}(t)
$$

Since MPR can influence the mean value of the asset, the value of asset satisfies the following processes:

$$
\begin{aligned}
d \delta(t)= & \theta_{\delta}\left(\mu_{\delta}-\delta(t)\right) d t+\theta_{\psi}\left(\mu_{\psi}-\psi(t)\right) d t \\
& -\left(\sigma_{\delta 1} v_{1}(t)+\sigma_{\delta 2} v_{2}(t)+\sigma_{\delta 3} v_{3}(t)\right) d t \\
& +\left(\sigma_{\delta 1} d \widehat{W}_{1}(t)+\sigma_{\delta 2} d \widehat{W}_{2}(t)+\sigma_{\delta 3} d \widehat{W}_{3}(t)\right)
\end{aligned}
$$

where

$$
\begin{aligned}
d \psi(t)= & \theta_{\psi}\left(\mu_{\psi}-\psi(t)\right) d t-\left(\sigma_{\psi 1} v_{1}(t)+\sigma_{\psi 2} v_{2}(t)+\sigma_{\psi 3} v_{3}(t)\right) d t \\
& +\left(\sigma_{\psi 1} d \widehat{W}_{1}(t)+\sigma_{\psi 2} d \widehat{W}_{2}(t)+\sigma_{\psi 3} d \widehat{W}_{3}(t)\right)
\end{aligned}
$$

as Ornstein-Uhlenbeck process can describe market price of risk:

$$
d v_{i}(t)=\bar{\theta}_{v i}\left(\bar{\mu}_{v i}-v_{i}(t)\right) d t+\sigma_{v i} d \widehat{W}_{i}(t)
$$

where

$$
\begin{aligned}
& \bar{\theta}_{v i}=\theta_{v i}\left(1+\sigma_{v i}\right), \\
& \bar{\mu}_{v i}=\frac{\mu_{v i}}{1+\sigma_{v i}} .
\end{aligned}
$$

First, we evaluate the European call option to acquire a formula of European put option through the application of put-call parity concept. The European call option $C(t)$ can then be described as follows:

$$
C(t)=E^{Q}\left[e^{-r(T-t)}[S(T)-K]^{+} \mid F_{t}\right]
$$




$$
=e^{-r(T-t)} E^{Q}\left[S(T) \mathbf{1}_{S(T)>K} \mid F_{t}\right]-K e^{-r(T-t)} E^{Q}\left[\mathbf{1}_{S(T)>K} \mid F_{t}\right]
$$

when

$$
\mathbf{1}_{S(T)>K}= \begin{cases}1 & \text { if } S(T)>K \\ 0 & \text { if otherwise }\end{cases}
$$

where $T, K$, and $r$ are times to maturity, strike price of an option, and interest rate respectively.

It is noted that the stock price $S(T)$ can be defined as

$$
S(T)=S(t) \exp \left\{\left(r-\frac{\sigma_{S}^{2}}{2}(T-t)-\int_{t}^{T} \delta(s) d s+\sigma_{S} \int_{t}^{T} d \widehat{W}_{1}(S)\right\}\right.
$$

$\int_{t}^{T} \delta(s) d s$, which is the total dividend yield, can be modeled from (12) as follows:

$$
\begin{aligned}
\int_{t}^{T} \delta(s) d s & \\
= & \mu_{\delta}(T-t)-\frac{1}{\theta_{\delta}}(\delta(T)-\delta(t))+\frac{\theta_{\psi}}{\theta_{\delta}} \mu_{\psi}(T-t) \\
& \quad-\frac{1}{\theta_{\delta}} \int_{t}^{T} \psi(s) d s-\frac{1}{\theta_{\delta}} \int_{t}^{T}\left(\sigma_{\delta 1} \nu_{1}(t)+\sigma_{\delta 2} \nu_{2}(t)+\sigma_{\delta 3} \nu_{3}(t)\right) d s \\
& +\left(\frac{\sigma_{\delta 1}}{\theta_{\delta}} \int_{t}^{T} d \widehat{W}_{1}(s)+\frac{\sigma_{\delta 2}}{\theta_{\delta}} \int_{t}^{T} d \widehat{W}_{2}(s)+\frac{\sigma_{\delta 3}}{\theta_{\delta}} \int_{t}^{T} d \widehat{W}_{3}(s)\right) .
\end{aligned}
$$

Working on (21) to solve the equations by using analytical techniques, $\delta(T)$ can be attained as follows:

$$
\begin{aligned}
\delta(T)= & \delta(t) e^{-\theta_{\delta}(T-t)}+\mu_{\delta}\left(1-e^{-\theta_{\delta}(T-t)}\right)+\frac{\theta_{\psi} \mu_{\psi}}{\theta_{\delta}}\left(1-e^{-\theta_{\delta}(T-t)}\right) \\
& -\frac{\psi(t)}{\theta_{\delta}-\theta_{\psi}}\left(e^{-\theta_{\psi}(T-t)}-e^{-\theta_{\delta}(T-t)}\right)-\frac{\mu_{\psi}}{\theta_{\delta}-\theta_{\psi}}\left(1-e^{-\theta_{\psi}(T-t)}\right) \\
& +\frac{\theta_{\psi} \mu_{\psi}}{\theta_{\delta}}\left(1-e^{-\theta_{\delta}(T-t)}\right) \\
& -\frac{\sigma_{\delta 1} \bar{\mu}_{\nu 1}}{\theta_{\delta}-\bar{\theta}_{\nu 1}}\left(\frac{\bar{\theta}_{\nu 1}}{\theta_{\delta}}\left(1-e^{-\theta_{\delta}(T-t)}\right)-\left(1-e^{-\bar{\theta}_{\nu 1}(T-t)}\right)\right) \\
& +\frac{\sigma_{\psi 1} \bar{\mu}_{\nu 1}}{\left(\theta_{\delta}-\theta_{\psi}\right)\left(\theta_{\psi}-\bar{\theta}_{\nu 1}\right)}\left(\frac{\bar{\theta}_{\nu 1}}{\theta_{\psi}}\left(1-e^{-\theta_{\psi}(T-t)}\right)-\left(1-e^{-\bar{\theta}_{\nu 1}(T-t)}\right)\right) \\
& -\frac{\sigma_{\psi 1} \bar{\mu}_{\nu 1}}{\left(\theta_{\delta}-\theta_{\psi}\right)\left(\theta_{\delta}-\bar{\theta}_{\nu 1}\right)}\left(\frac{\bar{\theta}_{\nu 1}}{\theta_{\delta}}\left(1-e^{-\theta_{\psi}(T-t)}\right)-\left(1-e^{-\bar{\theta}_{\nu 1}(T-t)}\right)\right) \\
& -\frac{\sigma_{\delta 2} \bar{\mu}_{\nu 2}}{\theta_{\delta}-\bar{\theta}_{\nu 2}}\left(\frac{\bar{\theta}_{\nu 2}}{\theta_{\delta}}\left(1-e^{-\theta_{\delta}(T-t)}\right)-\left(1-e^{-\bar{\theta}_{\nu 2}(T-t)}\right)\right) \\
& +\frac{\sigma_{\psi 2} \bar{\mu}_{\nu 2}}{\left(\theta_{\delta}-\theta_{\psi}\right)\left(\theta_{\psi}-\bar{\theta}_{\nu 2}\right)}\left(\frac{\bar{\theta}_{\nu 2}}{\theta_{\psi}}\left(1-e^{-\theta_{\psi}(T-t)}\right)-\left(1-e^{-\bar{\theta}_{\nu 2}(T-t)}\right)\right) \\
& -\frac{\sigma_{\psi 2} \bar{\mu}_{\nu 2}}{\left(\theta_{\delta}-\theta_{\psi}\right)\left(\theta_{\delta}-\bar{\theta}_{\nu 2}\right)}\left(\frac{\bar{\theta}_{\nu 2}}{\theta_{\delta}}\left(1-e^{-\theta_{\psi}(T-t)}\right)-\left(1-e^{-\bar{\theta}_{\nu 2}(T-t)}\right)\right) \\
& \\
&
\end{aligned}
$$




$$
\begin{aligned}
& -\frac{\sigma_{\delta 3} \bar{\mu}_{\nu 3}}{\theta_{\delta}-\bar{\theta}_{\nu 3}}\left(\frac{\bar{\theta}_{\nu 3}}{\theta_{\delta}}\left(1-e^{-\theta_{\delta}(T-t)}\right)-\left(1-e^{-\bar{\theta}_{\nu 3}(T-t)}\right)\right) \\
& +\frac{\sigma_{\psi 3} \bar{\mu}_{\nu 3}}{\left(\theta_{\delta}-\theta_{\psi}\right)\left(\theta_{\psi}-\bar{\theta}_{\nu 3}\right)}\left(\frac{\bar{\theta}_{\nu 3}}{\theta_{\psi}}\left(1-e^{-\theta_{\psi}(T-t)}\right)-\left(1-e^{-\bar{\theta}_{\nu 3}(T-t)}\right)\right) \\
& -\frac{\sigma_{\psi 3} \bar{\mu}_{\nu 3}}{\left(\theta_{\delta}-\theta_{\psi}\right)\left(\theta_{\delta}-\bar{\theta}_{\nu 3}\right)}\left(\frac{\bar{\theta}_{\nu 3}}{\theta_{\delta}}\left(1-e^{-\theta_{\psi}(T-t)}\right)-\left(1-e^{-\bar{\theta}_{\nu 3}(T-t)}\right)\right) \\
& -\frac{\sigma_{\delta 1}}{\theta_{\delta}-\bar{\theta}_{\nu 1}}\left(e^{-\theta_{\delta}(T-t)}-e^{-\bar{\theta}_{\nu 1}(T-t)}\right) v_{1}(t) \\
& +\frac{\sigma_{\psi 1}}{\left(\theta_{\delta}-\theta_{\psi}\right)\left(\theta_{\psi}-\bar{\theta}_{\nu 1}\right)}\left(e^{-\theta_{\psi}(T-t)}-e^{-\bar{\theta}_{\nu 1}(T-t)}\right) \nu_{1}(t) \\
& +\frac{\sigma_{\psi 1}}{\left(\theta_{\delta}-\theta_{\psi}\right)\left(\theta_{\delta}-\bar{\theta}_{\nu 1}\right)}\left(e^{-\theta_{\delta}(T-t)}-e^{-\bar{\theta}_{\nu 1}(T-t)}\right) \nu_{1}(t) \\
& -\frac{\sigma_{\delta 2}}{\theta_{\delta}-\bar{\theta}_{\nu 2}}\left(e^{-\theta_{\delta}(T-t)}-e^{-\bar{\theta}_{\nu 2}(T-t)}\right) \nu_{2}(t) \\
& +\frac{\sigma_{\psi 2}}{\left(\theta_{\delta}-\theta_{\psi}\right)\left(\theta_{\psi}-\bar{\theta}_{\nu 2}\right)}\left(e^{-\theta_{\psi}(T-t)}-e^{-\bar{\theta}_{\nu 2}(T-t)}\right) \nu_{2}(t) \\
& +\frac{\sigma_{\psi 2}}{\left(\theta_{\delta}-\theta_{\psi}\right)\left(\theta_{\delta}-\bar{\theta}_{\nu 2}\right)}\left(e^{-\theta_{\delta}(T-t)}-e^{-\bar{\theta}_{\nu 2}(T-t)}\right) \nu_{2}(t) \\
& -\frac{\sigma_{\delta 3}}{\theta_{\delta}-\bar{\theta}_{\nu 3}}\left(e^{-\theta_{\delta}(T-t)}-e^{-\bar{\theta}_{\nu 3}(T-t)}\right) v_{3}(t) \\
& +\frac{\sigma_{\psi 3}}{\left(\theta_{\delta}-\theta_{\psi}\right)\left(\theta_{\psi}-\bar{\theta}_{\nu 3}\right)}\left(e^{-\theta_{\psi}(T-t)}-e^{-\bar{\theta}_{\nu 3}(T-t)}\right) \nu_{3}(t) \\
& +\frac{\sigma_{\psi 3}}{\left(\theta_{\delta}-\theta_{\psi}\right)\left(\theta_{\delta}-\bar{\theta}_{\nu 3}\right)}\left(e^{-\theta_{\delta}(T-t)}-e^{-\bar{\theta}_{\nu 1}(T-t)}\right) \nu_{3}(t) \\
& +\sigma_{\delta 1} \int_{t}^{T}\left(e^{-\theta_{\delta}(T-s)}-\frac{\sigma_{\nu 1}}{\theta_{\delta}-\bar{\theta}_{\nu 1}}\left(e^{-\theta_{\delta}(T-s)}-e^{-\bar{\theta}_{\nu 1}(T-s)}\right)\right) d \widehat{W}_{1}(s) \\
& -\frac{\sigma_{\psi 1}}{\theta_{\delta}-\theta_{\psi}} \int_{t}^{T}\left(e^{-\theta_{\psi}(T-s)}-\frac{\sigma_{\nu 1}}{\theta_{\delta}-\bar{\theta}_{\nu 1}}\left(e^{-\theta_{\psi}(T-s)}-e^{-\bar{\theta}_{\nu 1}(T-s)}\right)\right) d \widehat{W}_{1}(s) \\
& +\frac{\sigma_{\psi 1}}{\theta_{\delta}-\theta_{\psi}} \int_{t}^{T}\left(e^{-\theta_{\delta}(T-s)}-\frac{\sigma_{\nu 1}}{\theta_{\delta}-\bar{\theta}_{\nu 1}}\left(e^{-\theta_{\delta}(T-s)}-e^{-\bar{\theta}_{\nu 1}(T-s)}\right)\right) d \widehat{W}_{1}(s) \\
& +\sigma_{\delta 2} \int_{t}^{T}\left(e^{-\theta_{\delta}(T-s)}-\frac{\sigma_{\nu 1}}{\theta_{\delta}-\bar{\theta}_{\nu 2}}\left(e^{-\theta_{\delta}(T-s)}-e^{-\bar{\theta}_{\nu 2}(T-s)}\right)\right) d \widehat{W}_{2}(s) \\
& -\frac{\sigma_{\psi 2}}{\theta_{\delta}-\theta_{\psi}} \int_{t}^{T}\left(e^{-\theta_{\psi}(T-s)}-\frac{\sigma_{\nu 2}}{\theta_{\delta}-\bar{\theta}_{\nu 2}}\left(e^{-\theta_{\psi}(T-s)}-e^{-\bar{\theta}_{\nu 2}(T-s)}\right)\right) d \widehat{W}_{2}(s) \\
& +\frac{\sigma_{\psi 1}}{\theta_{\delta}-\theta_{\psi}} \int_{t}^{T}\left(e^{-\theta_{\delta}(T-s)}-\frac{\sigma_{\nu 2}}{\theta_{\delta}-\bar{\theta}_{\nu 2}}\left(e^{-\theta_{\delta}(T-s)}-e^{-\bar{\theta}_{\nu 2}(T-s)}\right)\right) d \widehat{W}_{1}(s) \\
& +\sigma_{\delta 3} \int_{t}^{T}\left(e^{-\theta_{\delta}(T-s)}-\frac{\sigma_{\nu 3}}{\theta_{\delta}-\bar{\theta}_{\nu 3}}\left(e^{-\theta_{\delta}(T-s)}-e^{-\bar{\theta}_{\nu 3}(T-s)}\right)\right) d \widehat{W}_{3}(s) \\
& -\frac{\sigma_{\psi 3}}{\theta_{\delta}-\theta_{\psi}} \int_{t}^{T}\left(e^{-\theta_{\psi}(T-s)}-\frac{\sigma_{\nu 3}}{\theta_{\delta}-\bar{\theta}_{\nu 3}}\left(e^{-\theta_{\psi}(T-s)}-e^{-\bar{\theta}_{\nu 3}(T-s)}\right)\right) d \widehat{W}_{3}(s) \\
& +\frac{\sigma_{\psi 3}}{\theta_{\delta}-\theta_{\psi}} \int_{t}^{T}\left(e^{-\theta_{\delta}(T-s)}-\frac{\sigma_{\nu 3}}{\theta_{\delta}-\bar{\theta}_{\nu 3}}\left(e^{-\theta_{\delta}(T-s)}-e^{-\bar{\theta}_{\nu 3}(T-s)}\right)\right) d \widehat{W}_{3}(s) .
\end{aligned}
$$


We can mathematically express the total earning yield $\int_{t}^{T} \psi(s) d s$ as follows:

$$
\begin{aligned}
& \int_{t}^{T} \psi(s) d s=\frac{1}{\theta_{\psi}} \psi(t)\left(1-e^{\theta_{\psi}(T-t)}\right)+\mu_{\psi}\left((T-t)-\frac{1}{\theta_{\psi}}\left(1-e^{-\theta_{\psi}(T-t)}\right)\right) \\
& -\frac{\sigma_{\psi 1} \bar{\mu}_{\nu 1}}{\theta_{\psi}}\left[(T-t)-\frac{1}{\bar{\theta}_{\nu 1}}\left(1-e^{-\bar{\theta}_{\nu 1}(T-t)}\right)\right. \\
& \left.-\frac{\bar{\theta}_{\nu 1}}{\theta_{\psi}\left(\theta_{\psi}-\bar{\theta}_{\nu 1}\right)}\left(1-e^{-\theta_{\psi}(T-t)}\right)+\frac{1}{\theta_{\psi}-\bar{\theta}_{\nu 1}}\left(1-e^{-\bar{\theta}_{\nu}(T-t)}\right)\right] \\
& -\frac{\sigma_{\psi 2} \bar{\mu}_{\nu 2}}{\theta_{\psi}}\left[(T-t)-\frac{1}{\bar{\theta}_{\nu 2}}\left(1-e^{-\bar{\theta}_{\nu 2}(T-t)}\right)\right. \\
& \left.-\frac{\bar{\theta}_{\nu 2}}{\theta_{\psi}\left(\theta_{\psi}-\bar{\theta}_{\nu 2}\right)}\left(1-e^{-\theta_{\psi}(T-t)}\right)+\frac{1}{\theta_{\psi}-\bar{\theta}_{\nu 2}}\left(1-e^{-\bar{\theta}_{\nu}(T-t)}\right)\right] \\
& -\frac{\sigma_{\psi 3} \bar{\mu}_{\nu 3}}{\theta_{\psi}}\left[(T-t)-\frac{1}{\bar{\theta}_{\nu 3}}\left(1-e^{-\bar{\theta}_{\nu 3}(T-t)}\right)\right. \\
& \left.-\frac{\bar{\theta}_{\nu 3}}{\theta_{\psi}\left(\theta_{\psi}-\bar{\theta}_{\nu 3}\right)}\left(1-e^{-\theta_{\psi}(T-t)}\right)+\frac{1}{\theta_{\psi}-\bar{\theta}_{\nu 3}}\left(1-e^{-\bar{\theta}_{\nu}(T-t)}\right)\right] \\
& +\frac{\sigma_{\psi 1}}{\theta_{\psi}}\left[\frac{1}{\theta_{\psi}-\bar{\theta}_{\nu 1}}\left(e^{-\theta_{\psi}(T-t)}-e^{-\bar{\theta}_{\nu 1}(T-t)}\right)-\frac{1}{\bar{\theta}_{\nu 1}}\left(1-e^{-\bar{\theta}_{\nu 1}(T-t)}\right)\right] v_{1}(t) \\
& +\frac{\sigma_{\psi 2} 2}{\theta_{\psi}}\left[\frac{1}{\theta_{\psi}-\bar{\theta}_{\nu 2}}\left(e^{-\theta_{\psi}(T-t)}-e^{-\bar{\theta}_{\nu 2}(T-t)}\right)-\frac{1}{\bar{\theta}_{\nu 2}}\left(1-e^{-\bar{\theta}_{\nu 2}(T-t)}\right)\right] v_{2}(t) \\
& +\frac{\sigma_{\psi 3}}{\theta_{\psi}}\left[\frac{1}{\theta_{\psi}-\bar{\theta}_{\nu 3}}\left(e^{-\theta_{\psi}(T-t)}-e^{-\bar{\theta}_{\nu 3}(T-t)}\right)-\frac{1}{\bar{\theta}_{\nu 3}}\left(1-e^{-\bar{\theta}_{\nu 3}(T-t)}\right)\right] \nu_{3}(t) \\
& +\frac{\sigma_{\psi 1}}{\theta_{\psi}} \int_{t}^{T}\left[1-\frac{\sigma_{\nu 1}}{\bar{\theta}_{\nu 1}}+\frac{\sigma_{\nu 1}}{\bar{\theta}_{\nu 1}} e^{-\bar{\theta}_{\nu 1}(T-s)}-e^{-\theta_{\psi}(T-s)}\right. \\
& \left.+\frac{\sigma_{\nu 1}}{\theta_{\psi}-\bar{\theta}_{\nu 1}}\left(e^{-\theta_{\psi}(T-s)}-e^{-\bar{\theta}_{\nu 1}(T-s)}\right)\right] d \widehat{W}_{1}(s) \\
& +\frac{\sigma_{\psi 2}}{\theta_{\psi}} \int_{t}^{T}\left[1-\frac{\sigma_{\nu 2}}{\bar{\theta}_{\nu 2}}+\frac{\sigma_{\nu 2}}{\bar{\theta}_{\nu 2}} e^{-\bar{\theta}_{\nu 2}(T-s)}-e^{-\theta_{\psi}(T-s)}\right. \\
& \left.+\frac{\sigma_{v 2}}{\theta_{\psi}-\bar{\theta}_{\nu 2}}\left(e^{-\theta_{\psi}(T-s)}-e^{-\bar{\theta}_{\nu 2}(T-s)}\right)\right] d \widehat{W}_{2}(s) \\
& +\frac{\sigma_{\psi 3}}{\theta_{\psi}} \int_{t}^{T}\left[1-\frac{\sigma_{\nu 3}}{\bar{\theta}_{\nu 3}}+\frac{\sigma_{\nu 3}}{\bar{\theta}_{\nu 3}} e^{-\bar{\theta}_{\nu 3}(T-s)}-e^{-\theta_{\psi}(T-s)}\right. \\
& \left.+\frac{\sigma_{\nu 3}}{\theta_{\psi}-\bar{\theta}_{\nu 3}}\left(e^{-\theta_{\psi}(T-s)}-e^{-\bar{\theta}_{\nu 3}(T-s)}\right)\right] d \widehat{W}_{3}(s) .
\end{aligned}
$$

The total MPR $\int_{t}^{T} v_{i}(s) d s$ is derived as follows:

$$
\begin{aligned}
\int_{t}^{T} \nu_{i}(s) d s= & \frac{\nu_{i}}{t}\left(1-e^{-\bar{\theta}_{v i}(T-t)}\right)+\bar{\mu}_{\nu i}(T-t)-\frac{\bar{\mu}_{\nu i}}{\bar{\theta}_{\nu i}}\left(1-e^{-\bar{\theta}_{\nu i}(T-t)}\right) \\
& +\int_{t}^{T}\left(\frac{\sigma_{\nu_{i}}}{\bar{\theta}_{\nu i}}-\frac{\sigma_{\nu} i}{\bar{\theta}_{v i}} e^{-\bar{\theta}_{\nu i}(T-s)}\right) d \widehat{W}_{i}(s) .
\end{aligned}
$$

By substitution, we can solve for $S(T)$ and develop the formula for stock pricing. 
The structure of stock distribution is important because it shows that our proposed stochastic parameters may be included when pricing the option for stocks. With this, we can form a new stock valuation model using the analytical approach based on the relationship speculated. The following is our proposition.

Proposition 1 The formula for stock price can be considered as

$$
\begin{aligned}
S(T)= & S(t) \exp \left\{\left(r-\frac{\sigma_{S}^{2}}{2}(T-t)-L_{0}(t) \delta(t)-L_{1}(t)-M_{0}(t) \psi(t)-M_{1}(t)+O(t)\right.\right. \\
& +\sigma_{\delta 1}\left(L_{2}(t)-L_{3}(t) \nu_{1}(t)\right)+\sigma_{\delta 2}\left(L_{4}(t)-L_{5}(t) \nu_{2}(t)\right) \\
& +\sigma_{\delta 3}\left(L_{6}(t)-L_{7}(t) \nu_{3}(t)\right) \\
& +\sigma_{\psi 1}\left(M_{2}(t)-M_{3}(t) \nu 1(t)\right)+\sigma_{\psi 2}\left(M_{4}(t)-M_{5}(t) \nu 2(t)\right) \\
& +\sigma_{\psi 3}\left(M_{6}(t)-M_{7}(t) \nu 3(t)\right) \\
& \left.+\int_{t}^{T} \varepsilon_{1}(s) d \widehat{W}_{1}(s)+\int_{t}^{T} \varepsilon_{2}(s) d \widehat{W}_{2}(s)+\int_{t}^{T} \varepsilon_{3}(s) d \widehat{W}_{3}(s)\right\},
\end{aligned}
$$

where $\widehat{W}_{i}(t)$ is the generalized Ornstein-Uhlenbeck Wiener process and $L_{i}(t), M_{i}(t), O(t)$, and $\varepsilon_{i}(s)$ are as follows:

$$
\begin{aligned}
L_{0}(t)= & \frac{1}{\theta_{\delta}}\left(1-e^{-\theta_{\delta}(T-t)}\right), \\
L_{1}(t)= & \mu_{\delta}\left[(T-t)-\frac{1}{\theta_{\delta}}\left(1-e^{-\theta_{\delta}(T-t)}\right)\right], \\
L_{2}(t)= & \frac{\bar{\mu}_{\nu 1}}{\theta_{\delta}}\left[(T-t)-\frac{1}{\bar{\theta}_{\nu 1}}\left(1-e^{-\bar{\theta}_{\nu 1}(T-t)}\right)\right. \\
& \left.-\frac{1}{\theta_{\delta}-\bar{\theta}_{\nu 1}}\left(\frac{\bar{\theta}_{\nu 1}}{\theta_{\delta}}\left(1-e^{-\theta_{\delta}(T-t)}\right)-\left(1-e^{-\bar{\theta}_{\nu 1}(T-t)}\right)\right)\right], \\
L_{3}(t)= & \frac{1}{\theta_{\delta}}\left[\frac{1}{\theta_{\delta}-\bar{\theta}_{\nu 1}}\left(e^{-\theta_{\delta}(T-t)}-e^{-\bar{\theta}_{\nu 1}(T-t)}\right)-\frac{1}{\bar{\theta}_{\nu 1}}\left(1-e^{-\bar{\theta}_{\nu 1}(T-t)}\right)\right], \\
L_{4}(t)= & \frac{\mu_{\nu 2}}{\theta_{\delta}}\left[(T-t)-\frac{1}{\bar{\theta}_{\nu 2}}\left(1-e^{-\bar{\theta}_{\nu 2}(T-t)}\right)\right. \\
& \left.-\frac{1}{\theta_{\delta}-\bar{\theta}_{\nu 2}}\left(\frac{\bar{\theta}_{\nu 2}}{\theta_{\delta}}\left(1-e^{-\theta_{\delta}(T-t)}\right)-\left(1-e^{-\theta_{\nu 2}(T-t)}\right)\right)\right], \\
L_{7}(t)= & \frac{1}{\theta_{\delta}}\left[\frac{1}{\theta_{\delta}-\bar{\theta}_{\nu 3}}\left(e^{-\theta_{\delta}(T-t)}-e^{-\bar{\theta}_{\nu 3}(T-t)}\right)-\frac{1}{\bar{\theta}_{\nu 3}}\left(1-e^{-\bar{\theta}_{\nu 3}(T-t)}\right)\right], \\
M_{0}(t)= & \frac{1}{\theta_{\delta}}\left[\frac{1}{\theta_{\delta}-\theta_{\psi}}\left(e^{-\theta_{\psi}(T-t)}-e^{-\theta_{\delta}(T-t)}\right)-\frac{1}{\theta_{\psi}}\left(1-e^{-\theta_{\psi}(T-t)}\right)\right], \\
L_{5}(t)= & \frac{1}{\theta_{\delta}}\left[\frac{1}{\theta_{\delta}-\bar{\theta}_{\nu 2}}\left(e^{-\theta_{\delta}(T-t)}-e^{-\bar{\theta}_{\nu 2}(T-t)}\right)-\frac{1}{\bar{\theta}_{\nu 2}}\left(1-e^{-\bar{\theta}_{\nu 2}(T-t)}\right)\right], \\
L_{6}(t)= & \frac{\bar{\mu}_{\nu 3}}{\theta_{\delta}}\left[(T-t)-\frac{1}{\bar{\theta}_{\nu 3}}\left(1-e^{-\bar{\theta}_{\nu 3}(T-t)}\right)\right. \\
& -\bar{\theta}_{\delta}(T-t) \\
& \\
&
\end{aligned}
$$


Phewchean and Wu Advances in Difference Equations

(2019) 2019:277

Page 10 of 15

$$
\begin{aligned}
& M_{1}(t)=\frac{\mu_{\psi}}{\theta_{\delta}}\left[\frac{1}{\theta_{\delta}-\theta_{\psi}}\left(1-e^{-\theta_{\psi}(T-t)}\right)-\frac{2 \theta_{\psi}}{\theta_{\delta}}\left(1-e^{-\theta_{\delta}(T-t)}\right)-(T-t)+\frac{1}{\theta_{\psi}}\left(1-e^{-\theta_{\psi}(T-t)}\right)\right], \\
& M_{2}(t)=\frac{\bar{\mu}_{\nu 1}}{\theta_{\delta}}\left[\frac{1}{\left(\theta_{\delta}-\theta_{\psi}\right)\left(\theta_{\psi}-\bar{\theta}_{\nu 1}\right)}\left(\frac{\bar{\theta}_{\nu 1}}{\theta_{\psi}}\left(1-e^{-\theta_{\psi}(T-t)}\right)-\left(1-e^{-\bar{\theta}_{\nu 1}(T-t)}\right)\right)\right. \\
& -\frac{1}{\left(\theta_{\delta}-\theta_{\psi}\right)\left(\theta_{\delta}-\bar{\theta}_{\nu 1}\right)}\left(\frac{\bar{\theta}_{\nu 1}}{\theta_{\delta}}\left(1-e^{-\theta_{\psi}(T-t)}\right)-\left(1-e^{-\bar{\theta}_{\nu 1}(T-t)}\right)\right) \\
& +\frac{1}{\theta_{\psi}}\left((T-t)-\frac{1}{\bar{\theta}_{\nu 1}}\left(1-e^{-\theta_{\nu 1}(T-t)}\right)-\frac{\bar{\theta}_{\nu 1}}{\theta_{\psi}\left(\theta_{\psi}-\bar{\theta}_{\nu 1}\right)}\left(1-e^{-\theta_{\psi}(T-t)}\right)\right) \\
& \left.+\frac{1}{\theta_{\psi}}\left(\frac{1}{\theta_{\psi}-\bar{\theta}_{\nu 1}}\left(1-e^{1-e^{-} \bar{\theta}_{\nu 1}(T-t)}\right)\right)\right] \text {, } \\
& M_{3}(t)=\frac{1}{\theta_{\delta}}\left[\frac{1}{\left(\theta_{\delta}-\theta_{\psi}\right)\left(\theta_{\delta}-\bar{\theta}_{\nu 1}\right)}\left(e^{-\theta_{\delta}(T-t)}-e^{-\theta_{\nu 1}(T-t)}\right)\right. \\
& -\frac{1}{\left(\theta_{\delta}-\theta_{\psi}\right)\left(\theta_{\psi}-\bar{\theta}_{\nu 1}\right)}\left(e^{-\theta_{\psi}(T-t)}-e^{-\theta_{\nu 1}(T-t)}\right) \\
& \left.-\frac{1}{\theta_{\psi}}\left(\frac{1}{\theta_{\psi}-\bar{\theta}_{\nu 1}}\left(e^{-\theta_{\psi}(T-t)}-e^{-\bar{\theta}_{\nu 1}(T-t)}\right)-\frac{1}{\bar{\theta}_{\nu 1}}\left(1-e^{-\bar{\theta}_{\nu 1}(T-t)}\right)\right)\right], \\
& M_{4}(t)=\frac{\bar{\mu}_{\nu 2}}{\theta_{\delta}}\left[\frac{1}{\left(\theta_{\delta}-\theta_{\psi}\right)\left(\theta_{\psi}-\bar{\theta}_{\nu 2}\right)}\left(\frac{\bar{\theta}_{\nu 2}}{\theta_{\psi}}\left(1-e^{-\theta_{\psi}(T-t)}\right)-\left(1-e^{-\bar{\theta}_{\nu 2}(T-t)}\right)\right)\right. \\
& -\frac{1}{\left(\theta_{\delta}-\theta_{\psi}\right)\left(\theta_{\delta}-\bar{\theta}_{\nu 2}\right)}\left(\frac{\bar{\theta}_{\nu 2}}{\theta_{\delta}}\left(1-e^{-\theta_{\psi}(T-t)}\right)-\left(1-e^{-\bar{\theta}_{\nu 2}(T-t)}\right)\right) \\
& +\frac{1}{\theta_{\psi}}\left((T-t)-\frac{1}{\bar{\theta}_{\nu 2}}\left(1-e^{-\theta_{\nu 2}(T-t)}\right)-\frac{\bar{\theta}_{\nu 2}}{\theta_{\psi}\left(\theta_{\psi}-\bar{\theta}_{\nu 2}\right)}\left(1-e^{-\theta_{\psi}(T-t)}\right)\right) \\
& \left.+\frac{1}{\theta_{\psi}}\left(\frac{1}{\theta_{\psi}-\bar{\theta}_{\nu 2}}\left(1-e^{1-e^{-} \bar{\theta}_{\nu 2}(T-t)}\right)\right)\right] \text {, } \\
& M_{5}(t)=\frac{1}{\theta_{\delta}}\left[\frac{1}{\left(\theta_{\delta}-\theta_{\psi}\right)\left(\theta_{\delta}-\bar{\theta}_{\nu 2}\right)}\left(e^{-\theta_{\delta}(T-t)}-e^{-\theta_{\nu 2}(T-t)}\right)\right. \\
& -\frac{1}{\left(\theta_{\delta}-\theta_{\psi}\right)\left(\theta_{\psi}-\bar{\theta}_{\nu 2}\right)}\left(e^{-\theta_{\psi}(T-t)}-e^{-\theta_{\nu 2}(T-t)}\right) \\
& \left.-\frac{1}{\theta_{\psi}}\left(\frac{1}{\theta_{\psi}-\bar{\theta}_{\nu 2}}\left(e^{-\theta_{\psi}(T-t)}-e^{-\bar{\theta}_{\nu 2}(T-t)}\right)-\frac{1}{\bar{\theta}_{\nu 2}}\left(1-e^{-\bar{\theta}_{\nu 2}(T-t)}\right)\right)\right], \\
& M_{6}(t)=\frac{\bar{\mu}_{\nu 1}}{\theta_{\delta}}\left[\frac{1}{\left(\theta_{\delta}-\theta_{\psi}\right)\left(\theta_{\psi}-\bar{\theta}_{\nu 3}\right)}\left(\frac{\bar{\theta}_{\nu 3}}{\theta_{\psi}}\left(1-e^{-\theta_{\psi}(T-t)}\right)-\left(1-e^{-\bar{\theta}_{\nu 3}(T-t)}\right)\right)\right. \\
& -\frac{1}{\left(\theta_{\delta}-\theta_{\psi}\right)\left(\theta_{\delta}-\bar{\theta}_{\nu 3}\right)}\left(\frac{\bar{\theta}_{\nu 3}}{\theta_{\delta}}\left(1-e^{-\theta_{\psi}(T-t)}\right)-\left(1-e^{-\bar{\theta}_{\nu 3}(T-t)}\right)\right) \\
& +\frac{1}{\theta_{\psi}}\left((T-t)-\frac{1}{\bar{\theta}_{\nu 3}}\left(1-e^{-\theta_{\nu 3}(T-t)}\right)-\frac{\bar{\theta}_{\nu 3}}{\theta_{\psi}\left(\theta_{\psi}-\bar{\theta}_{\nu 3}\right)}\left(1-e^{-\theta_{\psi}(T-t)}\right)\right) \\
& +\frac{1}{\theta_{\psi}}\left(\frac{1}{\theta_{\psi}-\bar{\theta}_{\nu 3}}\left(1-e^{\left.1-e^{-\bar{\theta}_{\nu 3}(T-t)}\right)}\right)\right] \text {, } \\
& M_{7}(t)=\frac{1}{\theta_{\delta}}\left[\frac{1}{\left(\theta_{\delta}-\theta_{\psi}\right)\left(\theta_{\delta}-\bar{\theta}_{\nu 3}\right)}\left(e^{-\theta_{\delta}(T-t)}-e^{-\theta_{\nu 3}(T-t)}\right)\right.
\end{aligned}
$$




$$
\begin{aligned}
& -\frac{1}{\left(\theta_{\delta}-\theta_{\psi}\right)\left(\theta_{\psi}-\bar{\theta}_{\nu 3}\right)}\left(e^{-\theta_{\psi}(T-t)}-e^{-\theta_{\nu 3}(T-t)}\right) \\
& \left.-\frac{1}{\theta_{\psi}}\left(\frac{1}{\theta_{\psi}-\bar{\theta}_{\nu 3}}\left(e^{-\theta_{\psi}(T-t)}-e^{-\bar{\theta}_{\nu 3}(T-t)}\right)-\frac{1}{\bar{\theta}_{\nu 3}}\left(1-e^{-\bar{\theta}_{\nu 3}(T-t)}\right)\right)\right], \\
& O(t)=\frac{\theta_{\psi}}{\theta_{\delta}}(T-t) \\
& \varepsilon_{1}(s)=\sigma_{S}-\left[\left(\frac{\sigma_{\delta 1}}{\theta_{\delta}}-\frac{\sigma_{\delta 1} \sigma_{\nu 1}}{\theta_{\delta} \bar{\theta}_{\nu 1}}-\frac{\sigma_{\psi 1}}{\theta_{\psi} \theta_{\delta}}+\frac{\sigma_{\psi 1} \sigma_{\nu 1}}{\theta_{\psi} \theta_{\delta} \bar{\theta}_{\nu 1}}\right)\right. \\
& +\left(\frac{\sigma_{\delta 1} \sigma_{v 1}}{\theta_{\delta} \bar{\theta}_{\nu 1}}-\frac{\sigma_{\delta 1} \sigma_{v 1}}{\theta_{\delta}\left(\theta_{\delta}-\bar{\theta}_{v 1}\right)}-\frac{\sigma_{\psi 1} \sigma_{v 1}}{\theta_{\psi} \theta_{\delta} \bar{\theta}_{\nu 1}}+\frac{\sigma_{\psi 1} \sigma_{\nu 1}}{\theta_{\psi} \theta_{\delta}\left(\theta_{\psi}-\bar{\theta}_{\nu 1}\right)}\right) e^{-\bar{\theta}_{\nu 1}(T-s)} \\
& +\left(\frac{\sigma_{\delta 1} \sigma_{\nu 1}}{\theta_{\delta}\left(\theta_{\delta}-\bar{\theta}_{\nu 1}\right)}-\frac{\sigma_{\delta 1}}{\theta_{\delta}}-\frac{\sigma_{\psi 1}}{\theta_{\delta}\left(\theta_{\delta}-\theta_{\psi}\right)}+\frac{\sigma_{\psi 1} \sigma_{\nu 1}}{\theta_{\delta}\left(\theta_{\delta}-\theta_{\psi}\right)\left(\theta_{\delta}-\bar{\theta}_{\nu 1}\right)}\right) e^{-\theta_{\delta}(T-s)} \\
& \left.+\left(\frac{\sigma_{\psi 1}}{\theta_{\delta}\left(\theta_{\delta}-\theta_{\psi}\right)}-\frac{\sigma_{\psi 1} \sigma_{\nu 1}}{\theta_{\delta}\left(\theta_{\delta}-\theta_{\psi}\right)\left(\theta_{\delta}-\bar{\theta}_{\nu 1}\right)}+\frac{\sigma_{\psi 1}}{\theta_{\psi} \theta_{\delta}}-\frac{\sigma_{\psi 1} \sigma_{\nu 1}}{\theta_{\psi} \theta_{\delta}\left(\theta_{\psi}-\bar{\theta}_{\nu 1}\right)}\right) e^{-\theta_{\psi}(T-s)}\right], \\
& \varepsilon_{2}(s)=-\left[\left(\frac{\sigma_{\delta 2}}{\theta_{\delta}}-\frac{\sigma_{\delta 2} \sigma_{\nu 2}}{\theta_{\delta} \bar{\theta}_{\nu 2}}-\frac{\sigma_{\psi 2}}{\theta_{\psi} \theta_{\delta}}+\frac{\sigma_{\psi 2} \sigma_{\nu 2}}{\theta_{\psi} \theta_{\delta} \bar{\theta}_{\nu 2}}\right)\right. \\
& +\left(\frac{\sigma_{\delta 2} \sigma_{\nu 2}}{\theta_{\delta} \bar{\theta}_{\nu 2}}-\frac{\sigma_{\delta 2} \sigma_{v 2}}{\theta_{\delta}\left(\theta_{\delta}-\bar{\theta}_{\nu 2}\right)}-\frac{\sigma_{\psi 2} \sigma_{\nu 2}}{\theta_{\psi} \theta_{\delta} \bar{\theta}_{\nu 2}}+\frac{\sigma_{\psi 2} \sigma_{v 2}}{\theta_{\psi} \theta_{\delta}\left(\theta_{\psi}-\bar{\theta}_{\nu 2}\right)}\right) e^{-\bar{\theta}_{\nu 2}(T-s)} \\
& +\left(\frac{\sigma_{\delta 2} \sigma_{\nu 2}}{\theta_{\delta}\left(\theta_{\delta}-\bar{\theta}_{\nu 2}\right)}-\frac{\sigma_{\delta 2}}{\theta_{\delta}}-\frac{\sigma_{\psi 2}}{\theta_{\delta}\left(\theta_{\delta}-\theta_{\psi}\right)}+\frac{\sigma_{\psi 2} \sigma_{\nu 2}}{\theta_{\delta}\left(\theta_{\delta}-\theta_{\psi}\right)\left(\theta_{\delta}-\bar{\theta}_{\nu 2}\right)}\right) e^{-\theta_{\delta}(T-s)} \\
& \left.+\left(\frac{\sigma_{\psi 2}}{\theta_{\delta}\left(\theta_{\delta}-\theta_{\psi}\right)}-\frac{\sigma_{\psi 2} \sigma_{\nu 2}}{\theta_{\delta}\left(\theta_{\delta}-\theta_{\psi}\right)\left(\theta_{\delta}-\bar{\theta}_{\nu 2}\right)}+\frac{\sigma_{\psi 2}}{\theta_{\psi} \theta_{\delta}}-\frac{\sigma_{\psi 2} \sigma_{\nu 2}}{\theta_{\psi} \theta_{\delta}\left(\theta_{\psi}-\bar{\theta}_{\nu 1}\right)}\right) e^{-\theta_{\psi}(T-s)}\right] \text {, } \\
& \varepsilon_{3}(s)=-\left[\left(\frac{\sigma_{\delta 3}}{\theta_{\delta}}-\frac{\sigma_{\delta 3} \sigma_{\nu 3}}{\theta_{\delta} \bar{\theta}_{\nu 3}}-\frac{\sigma_{\psi 3}}{\theta_{\psi} \theta_{\delta}}+\frac{\sigma_{\psi 3} \sigma_{\nu 3}}{\theta_{\psi} \theta_{\delta} \bar{\theta}_{\nu 3}}\right)\right. \\
& +\left(\frac{\sigma_{\delta 3} \sigma_{\nu 3}}{\theta_{\delta} \bar{\theta}_{\nu 3}}-\frac{\sigma_{\delta 3} \sigma_{\nu 3}}{\theta_{\delta}\left(\theta_{\delta}-\bar{\theta}_{\nu 3}\right)}-\frac{\sigma_{\psi 3} \sigma_{\nu 3}}{\theta_{\psi} \theta_{\delta} \bar{\theta}_{\nu 3}}+\frac{\sigma_{\psi 1} \sigma_{\nu 3}}{\theta_{\psi} \theta_{\delta}\left(\theta_{\psi}-\bar{\theta}_{\nu 3}\right)}\right) e^{-\bar{\theta}_{\nu 3}(T-s)} \\
& +\left(\frac{\sigma_{\delta 3} \sigma_{\nu 3}}{\theta_{\delta}\left(\theta_{\delta}-\bar{\theta}_{\nu 3}\right)}-\frac{\sigma_{\delta 3}}{\theta_{\delta}}-\frac{\sigma_{\psi 3}}{\theta_{\delta}\left(\theta_{\delta}-\theta_{\psi}\right)}+\frac{\sigma_{\psi 3} \sigma_{\nu 1}}{\theta_{\delta}\left(\theta_{\delta}-\theta_{\psi}\right)\left(\theta_{\delta}-\bar{\theta}_{\nu 3}\right)}\right) e^{-\theta_{\delta}(T-s)} \\
& \left.+\left(\frac{\sigma_{\psi 3}}{\theta_{\delta}\left(\theta_{\delta}-\theta_{\psi}\right)}-\frac{\sigma_{\psi 3} \sigma_{\nu 1}}{\theta_{\delta}\left(\theta_{\delta}-\theta_{\psi}\right)\left(\theta_{\delta}-\bar{\theta}_{\nu 3}\right)}+\frac{\sigma_{\psi 3}}{\theta_{\psi} \theta_{\delta}}-\frac{\sigma_{\psi 3} \sigma_{\nu 3}}{\theta_{\psi} \theta_{\delta}\left(\theta_{\psi}-\bar{\theta}_{\nu 3}\right)}\right) e^{-\theta_{\psi}(T-s)}\right] \text {. }
\end{aligned}
$$

We apply Ito's calculus and explicit computation to the proposition above as we develop the formulae for European options pricing.

\section{The options pricing formula}

From the preceding section, we provide definition for the specified Brownian process component to resolve the European options formula as follows:

$$
\Sigma(s)^{2}=\int_{t}^{T} \varepsilon_{1}(s)^{2} d \widehat{W}_{1}(s)+\int_{t}^{T} \varepsilon_{2}(s)^{2} d \widehat{W}_{2}(s)+\int_{t}^{T} \varepsilon_{3}(s)^{2} d \widehat{W}_{3}(s) .
$$

From (18), solve for $E^{Q}\left[S(T) \mathbf{1}_{S(T)>K} \mid F_{t}\right]$ and $E^{Q}\left[\mathbf{1}_{S(T)>K} \mid F_{t}\right]$ by given

$$
\Phi(t)=L_{0}(t) \delta(t)+L_{1}(t)+M_{0}(t) \psi(t)+M_{1}(t)+O(t)
$$




$$
\begin{aligned}
& -\sigma_{\delta 1}\left(L_{2}(t)-L_{3}(t) v_{1}(t)\right)-\sigma_{\delta_{2}}\left(L_{4}(t)-L_{5}(t) v_{2}(t)\right)-\sigma_{\delta_{3}}\left(L_{6}(t)-L_{7}(t) v_{3}(t)\right) \\
& -\sigma_{\psi 1}\left(M_{2}(t)-M_{3}(t) v_{1}(t)\right)-\sigma_{\psi 2}\left(M_{4}(t)-M_{5}(t) v_{2}(t)\right) \\
& -\sigma_{\psi 3}\left(M_{6}(t)-M_{7}(t) v_{3}(t)\right) .
\end{aligned}
$$

From (24) when $S(t)=K, \eta$ is defined as

$$
\eta \leq d_{2}=\frac{1}{\Sigma(t)}\left(\ln \frac{S(t)}{K}+\left(r-\frac{\sigma_{s}^{2}}{2}\right)(T-t)-\Phi(t)\right) .
$$

When $\eta$ is assumed to be under normal distribution, then we get

$$
E^{Q}\left[\mathbf{1}_{S(T)>K} \mid F_{t}\right]=N\left(d_{2}\right),
$$

where $d_{2}=\frac{1}{\Sigma(t)}\left(\ln \frac{S(t)}{K}+\left(r-\frac{\sigma_{s}^{2}}{2}\right)(T-t)-\Phi(t)\right)$.

$$
\begin{aligned}
E^{Q}\left[S(T) \mathbf{1}_{S(T)>K} \mid F_{t}\right] & =E^{Q}\left[S(T) \mathbf{1}_{\eta>-d_{2}} \mid F_{t}\right] \\
& =S(t) \exp \left\{\left(r-\frac{\sigma_{s}^{2}}{2}\right)(T-t)-\Phi(t)\right\} E^{Q}\left[e^{\Sigma(t) \eta} \mathbf{1}_{\eta>-d_{2}} \mid F_{t}\right] \\
& =S(t) \exp \left\{\left(r-\frac{\sigma_{s}^{2}}{2}\right)(T-t)-\Phi(t)\right\} e^{\frac{1}{2} \Sigma(t)^{2}} N\left(d_{1}\right)
\end{aligned}
$$

when $d_{1}=d_{2}+\Sigma(t)$.

With an application of (25) to (31), the formulas for European options are proposed as follows.

\subsection{European call option}

Under an environment of stochastic dividend yield $\delta(t)$ and stochastic earning yield $\psi(t)$, European call option $C(t)$ is formulated as follows.

Proposition 2 European call option $C$ with stochastic earning yield $\psi$ and stochastic dividend $\delta$ is formulated as

$$
C(t)=S(t) e^{\left[-\Phi(t)-\frac{\sigma_{S}^{2}}{2}(T-t)-\frac{1}{2} \Sigma(t)^{2}\right]} N\left(d_{1}\right)-K e^{-r(T-t)} N\left(d_{2}\right),
$$

where $d_{1}, d_{2}, \Phi(t), L_{0}(t)-L_{7}(t), M_{0}(t)-M_{7}(t)$, and $O(t)$ are given.

\subsection{Put-call parity}

Put-call parity for a stock, in financial mathematics, is a relationship between European call and put option. It is assumed that the price is a frictionless market. Under an assumption of arbitrage opportunities, the relationship between call and put option can be derived as follows:

$$
C(t)-P(t)=e^{-r(T-t)} E^{Q}\left[S(T)-K \mid F_{t}\right],
$$

where

$$
E^{Q}\left[S(T) \mid F_{t}\right]=S(t) \exp \left\{\left(r-\frac{\sigma_{s}^{2}}{2}\right)(T-t)-\Phi(t)\right\} E^{Q}\left[e^{\Sigma(t) \eta} \mid F_{t}\right]
$$




$$
=S(t) \exp \left\{\left(r-\frac{\sigma_{s}^{2}}{2}\right)(T-t)-\Phi(t)+\frac{1}{2} \Sigma(t)^{2}\right\} .
$$

The formula of put-call parity, following the conditions of stochastic dividend yield and stochastic earning yield, can be derived as follows.

Proposition 3 The put-call parity with stochastic earning yield $\psi(t)$ and stochastic dividend yield $\delta(t)$ is

$$
C(t)-P(t)=S(t) e^{\left[-\Phi(t)-\frac{\sigma_{S}^{2}}{2}(T-t)-\frac{1}{2} \Sigma(t)^{2}\right]}-K e^{-r(T-t)},
$$

where $\Phi(t), L_{0}(t)-L_{7}(t), M_{0}(t)-M_{7}(t)$, and $O(t)$ are given.

\subsection{European put option}

We can calculate the European put option formula $P(t)$ from Propositions 2 and 3. Provided as such, we can now determine an explicit formula for put option taking into account our proposed parameters.

Proposition 4 European put option $P(t)$ with stochastic earning yield $\psi$ and stochastic dividend $\delta$ is

$$
P(t)=K e^{-r(T-t)} N\left(-d_{2}\right)-S(t) e^{\left[-\Phi(t)-\frac{\sigma_{S}^{2}}{2}(T-t)-\frac{1}{2} \Sigma(t)^{2}\right]} N\left(-d_{1}\right),
$$

where $d_{1}, d_{2}, \Phi(t), L_{0}(t)-L_{7}(t), M_{0}(t)-M_{7}(t)$, and $O(t)$ are given (as detailed above and in the previous section).

With the component of stochastic dividend yield and stochastic earning yield as important stochastic factors in real situations along with the market price of risk (MPR), we can come up with four different models for the call option price (32), put-call parity relationship (36), and put option price (37).

\section{The simulation}

The simulation is executed through the use of data that exists in the real world to check the model consistency. The four models to be examined are the Black-Scholes model (BSC), the Black-Scholes-Merton model with constant dividend yield (CDC), the BlackScholes-Merton model with stochastic dividend yield (SDC), and the Black-ScholesMerton model with stochastic earning yield (SEC). The models are analyzed by measuring the mean squared errors. Hypothetically, small squared error means high consistency between the option pricing model and the data. Hence, the smaller the squared error, the greater the consistency.

As we apply our model to the European option, four stock indexes are included in our simulation. There are 1/100 Dow Jones Industrial Average, Standard and Poor 500, Russell 2000 and NASDAQ-100 as of the information from 2012 to 2017. Table 1 to Table 4 show the results of mean squared errors of the simulation.

According to our simulation, we can conclude that our proposed option pricing model, which considers the stochastic characteristics of dividend yield, earning yield, and market price of risk, is preeminent among the others. This means that our model is better at 
Table 1 Mean squared errors of option prices obtained by four different models for the 1/100 Dow Jones Industrial Average option prices

\begin{tabular}{llrrr}
\hline Model & BSC & CDC & SDC & \multicolumn{1}{c}{ SEC } \\
\hline Call options & 40.2322 & 38.9655 & 39.4315 & 19.4551 \\
Put options & 8.1210 & 7.9447 & 8.0101 & 5.7794 \\
\hline
\end{tabular}

Table 2 Mean squared errors of call option prices obtained by four different models for the Standard and Poor 500 option prices

\begin{tabular}{lrrrr}
\hline Model & BSC & CDC & SDC & \multicolumn{1}{c}{ SEC } \\
\hline Call options & 33.2921 & 28.7806 & 28.1293 & 21.7287 \\
Put options & 756.4057 & 652.3046 & 717.5044 & 552.8234 \\
\hline
\end{tabular}

Table 3 Mean squared errors of call option prices obtained by four different models for the Russell 2000 option prices

\begin{tabular}{llllr}
\hline Model & BSC & CDC & SDC & \multicolumn{1}{l}{ SEC } \\
\hline Call options & 6395.5434 & 5399.4494 & 6067.9173 & 2592.8771 \\
Put options & 1663.2853 & 1430.8668 & 1586.2697 & 361.0587 \\
\hline
\end{tabular}

Table 4 Mean squared errors of call option prices obtained by four different models for the NASDAQ-100 option prices

\begin{tabular}{lllll}
\hline Model & BSC & CDC & SDC & SEC \\
\hline Call options & 2874.4643 & 2229.2508 & 4901.8061 & 214.9527 \\
Put options & 4218.2402 & 4051.7451 & 4356.3951 & 440.1807 \\
\hline
\end{tabular}

enhancing the performance to valuate the fair price of option and improving the decisionmaking process of the investors.

\section{The conclusion}

The data available in newspapers, apart from the dividend yield, is the P/E ratio that is commonly used to aid investing decision. Considering finance fundamental concepts, it is possible that the earning yield, which is a reciprocal of the $\mathrm{P} / \mathrm{E}$ ratio, has an impact on the pricing model. A generalized Ornstein-Uhlenbeck process is examined to mathematically model the option price driven by the earning yield. The market price of risk is also defined as a part of the generalized Ornstein-Uhlenbeck process due to the complex movements of market risks. Further investigation leads to four propositions: the new stock pricing structure, the European call option formula, the put-call parity relationship, and the European put option formula are proposed. Then, we inspect our proposed models by comparing their outputs to the real-world market data. The data are selected from the source attribution on European option investments. Therefore, we use four stock indexes, namely 1/100 Dow Jones Industrial Average, Standard and Poor 500, Russell 2000, and NASDAQ-100. The simulation resulted in strong agreement between the models calculation and the data acquired in the recent market. This works for call and put option which is shown from the smallest mean of sum of squared errors in all four stock indexes. Our proposed option pricing model with an extension of the relevant stochastic factors, in essence, yields the most accurate values compared to three other existing models.

To conclude, our mathematical option pricing model that includes the stochastic earning yield, stochastic market price of risk, and stochastic earning yield has the highest po- 
tential in illustrating the financial market in recent world. Thus, for investor, the proposed model may be applied for a better result of option valuation in the market of financial world.

\section{Acknowledgements}

The authors would like to greatly thank the referees for their valuable suggestions and comments.

\section{Funding}

We acknowledge the support of the Centre of Excellence in Mathematics, CHE, Bangkok, Thailand.

\section{Availability of data and materials}

Data and material are publicly available at https://finance.yahoo.com/ and http://www.cboe.com/.

\section{Competing interests}

The authors declare that they have no competing interests.

\section{Consent for publication}

All authors have seen and approved the submission of this manuscript.

\section{Authors' contributions}

All authors contributed equally to this work. All authors read and approved the final manuscript.

\section{Author details}

${ }^{1}$ Department of Mathematics, Faculty of Science, Mahidol University, Bangkok, Thailand. ${ }^{2}$ Centre of Excellence in

Mathematics, CHE, Bangkok, Thailand. ${ }^{3}$ Department of Mathematics and Statistics, Curtin University, Perth, Australia.

\section{Publisher's Note}

Springer Nature remains neutral with regard to jurisdictional claims in published maps and institutional affiliations.

Received: 7 February 2019 Accepted: 20 June 2019 Published online: 08 July 2019

\section{References}

1. Black, F., Scholes, M.: The pricing of options and corporate liabilities. J. Polit. Econ. 81, 637-654 (1973)

2. Geske, R.: The pricing of options with stochastic dividend yield. J. Finance 12, 617-625 (1978)

3. Broadie, M., Detemple, J., Ghysels, E., Torres, O.: American options with stochastic dividends and volatility: a non parametric investigation. J. Econom. 94, 53-92 (2000)

4. Chance, D., Kumar, R., Rich, D.: European option pricing with discrete stochastic dividend. J. Deriv. 12, $39-45$ (1978)

5. Lioui, A.: Black-Scholes-Merton revisited under stochastic dividend yields. J. Futures Mark. 26, 703-732 (2006)

6. Heston, S.L., Nandi, S.: A closed-form GARCH option valuation model. Rev. Financ. Stud. 13, 585-625 (2000)

7. Hilliard, J.E., Reis, J.: Valuation of commodity futures and options under stochastic convenience yields, interest rates, and jump diffusions in the spot. J. Finance 12,617-625 (1978)

8. Cox, J.C., Ross, S.A.: The valuation of options for alternative stochastic processes. J. Financ. Econ. 3, 145-166 (1976)

9. Uhlenbeck, G.E., Ornstein, L.S.: On the theory of Brownian motion. Phys. Rev. 36, 823-841 (1930)

10. Harrison, J.M., Pliska, S.: Martingales and stochastic integrals in the theory of continuous trading. Stoch. Process. Appl. $11,215-260(1981)$

11. Kishimoto, M.: On the Black-Scholes Equation: Various Derivations. Springer, Berlin (1991)

12. Karatzas, I., Shreve, S.E.: Brownian Motion and Stochastic Calculus. Springer, Berlin (1991)

13. Kwok, Y.K.: Mathematical Models of Financial Derivatives. Springer, Berlin (1998)

\section{Submit your manuscript to a SpringerOpen ${ }^{\circ}$ journal and benefit from:}

- Convenient online submission

- Rigorous peer review

- Open access: articles freely available online

- High visibility within the field

- Retaining the copyright to your article 